\title{
A SURVEY ON SOFT COMPUTING TECHNIQUES USED IN INTELLIGENT BUILDING CONTROL
}

\author{
Tibor VESSELÉNYI \\ University of Oradea, \\ Faculty of Management \\ and Technological \\ Engineering \\ Oradea, Romania \\ tvesselenyi@yahoo.co.uk
}

\author{
Ovidiu MOLDOVAN \\ University of Oradea, \\ Faculty of Management \\ and Technological \\ Engineering \\ Oradea, Romania \\ ovidiu30@gmail.com
}

\author{
Constantin BUNG $\breve{A U}$ \\ University of Oradea, \\ Faculty of Management \\ and Technological \\ Engineering \\ Oradea, Romania \\ bungau@uoradea.ro
}

\author{
Lehel CSOKMAI \\ University of Oradea, \\ Faculty of Management \\ and Technological \\ Engineering \\ Oradea, Romania \\ cslehel@gmail.com
}

\begin{abstract}
In this paper, we present different papers discussing soft computing methods applied in development, optimization and control of intelligent building systems. We present the approaches used, their advantages in solving control problems, analyze and modelling of human behavior in order to implement energy saving control solutions besides accounting for user comfort.
\end{abstract}

Keywords- intelligent buildings, control methods, soft computing, inhabitant behavior.

\section{INTELLIGENT BUILDING CONTROL}

The concept of intelligent building has been established in the early 1980s, when this concept was mainly linked to building automation. Since then the concept has evolved to the point when a building is not considered as a collection of passive objects but an intelligent system which responds to the user's demands. In time a lot of other names had been used, to cover this field or parts of it, with some nuances: smart home, ambient ecologies or ubiquitous computing environments.

According to [1] an intelligent building should be a dynamic and responsive building that provides cost effective operation environment by optimizing materials, automation, maintenance and performance and their interrelations. The objectives of developing intelligent buildings are to create a highly energy efficient and environmental-friendly environment with substantial safety, security, well-being and convenience, a lower life-cycle cost, and long term flexibility and marketability.As it is shown in [1], 'building intelligence' has been employed as a unique term of measure to reflect the specific performance and properties of the intelligent building (IB). Researchers in this field developed indicators which enable the IB performance to be quantified in terms of the building systems installed and the level of integration that exists between them or in terms of building characteristics: site, cost, architecture, technology, responsiveness, access and security. Researchers in [1] proposed the analytic network process, a systemic analytical approach, to prioritize the intelligent indicators and develop the model for computing the system intelligent score - a measurement of the system intelligence of the intelligent building systems. Summarizing the results of these studies we can derive two of the most important factors which are the subject of study and goal in IB research: energy consumption and user contempt. In many cases these factors are the main topics of analysis, while trying to answer questions like: how to reduce energy consumption costs without diminishing user comfort, or how to raise user comfort without increasing energy consumption. In order to answer these questions researchers have to find innovative, energy saving solutions by either harvesting new energy source, using intelligent device or optimizing energy consumption control strategies. As an example of intelligent devices we can mention electrochromic windows that can cut total commercial building electricity peak load by 15 to 20 percent, and can make windows a net energy saver by increasing solar heat when needed, and rejecting it when not wanted. Another example is a new class of intelligent building controls that are enabling large cost-effective virtual storage at building levels. The best of these are able to incorporate past, current, and projected future temperature projections in designing lowest-energy-use strategies to achieve desired comfort [1]. As energy control of buildings is a complex multi - criteria optimization problem, usual control strategies can be applied with limited success. This is further complicated by the intricate problems of human comfort needs, which must not be forgotten if a truly intelligent building is to be developed. Summarizing we can say that an important challenge in building control is that the energy consumption and the comfort level in a building environment often conflict with each other. These kinds of problems relates to human subjectivity, for example as in the case of determining a 
suitable temperature for a room to make people feel comfortable. As a base to analyze human behavior related to Intelligent Building some researchers conducted studies of user activity. In [2] the authors concluded that inhabitant's decisions and actions have a strong impact on the energy consumption and are an important factor in reducing energy consumption and in modelling future energy trends. Energy simulations that take into account inhabitants' behavior are benchmarked at office buildings using controlled activity profiles and predefined scenarios. In their paper the researchers had proposed a co-simulation environment for energy smart homes that takes into account inhabitants' dynamic and social behavior. Based on this kind of complex behavior, the set points for different controllers had been adjusted in the physical simulator. In this study, a model of human behavior was developed. The thermal model computes the temperature decrease/increase in a room based on the contextual information resulting from the behavior simulator. This information is then given to the controller which adjusts the temperature in the room. In [3] the researchers present the implementation of a multi-agent comfort and energy system which models control of building systems and occupants. This system coordinates both building system devices and building occupants through direct changes to occupant meeting schedules using multi-objective Markov Decision Problems. The operations of this building are then simulated according to three distinct control strategies involving varying levels of intelligent coordination of devices and occupants. A $12 \%$ reduction in energy consumption and a 5\% improvement in occupant comfort are reportedly realized as compared to the baseline control.

In the same topic, user behavior detection framework based on non-parametric belief propagation (NBP) is presented in [4] and in [5] the authors made a survey of prominent intelligent buildings research efforts with the theme of energy saving and user activity recognition.

\section{SOFT COMPUTING CONTROL METHODS}

In matters which involve human behavior and interaction with machines an obvious choice is to use soft computing techniques which had been also proved to be successful in multi-criteria control strategies.

Soft computing techniques can be conventionally divided in the following categories:

- Artificial neural networks: Perceptron, Support Vector Machines - learning, modeling a system's behavior, adjusting parameters;

- $\quad$ Fuzzy logic - decision making, control;

- Evolutionary computation: Evolutionary algorithms, Genetic algorithms optimization;

- Swarm Intelligence: Ant colony, Particle swarm - optimization;
From this list of soft computing we found that artificial neural networks, fuzzy logic, and particle swarm optimization are mostly used in the field of our interest.

Conducting a query in main scientific paper databases (Elsevier, Springer) we found that research articles in the period 2010-2013, in the field of IB for:

- $35 \%-40 \%$ are dealing with soft computing control techniques;

- More than $50 \%$ are considering human behavior a major factor;

Most of the studies in Intelligent Building concluded that usual control methods like Proportional - Integrator Derivative (PID) or Model Predictive Control (MPC) alone cannot fulfil the goals of Intelligent Building control. This can be successfully used though, if they are combined with different soft computing methods as for example Artificial Neural Networks (ANN). In the following paragraph we present an example of a neural MPC method. MPC is a strategy of advanced control representing a vast class of control methods using a process model and minimizing an objective function subject to some constraints [6]. The minimization is performed in an iterative manner on a finite optimization horizon to predict a number of steps of control signal. In order to avoid feedback, robustness and stability problems a receding horizon is applied which means that the prediction horizon keeps being shifted forward (MPC receding prediction horizon, Soloway \& Haley, 1996). This have certain advantages in intelligent building control which is a complicated task as each building is unique and requires its own mathematical description.

\subsection{Artificial Neural Networks}

Identification phase. Figure 1. presents the identification process structure in which the prediction errors, defined as difference between process and neural model outputs are used as training signals and figure 2. presents the neural network which models the process and uses previous inputs and outputs to generate control signals [12].

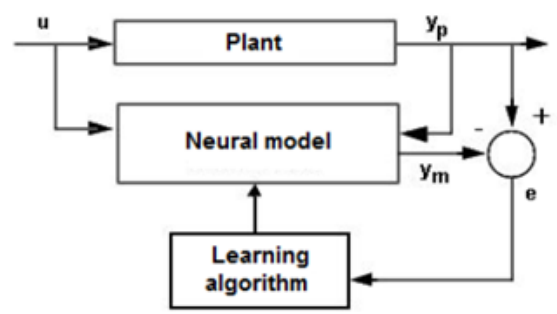

Fig.1. Process neural model identification.

Notations in figure 1 are: $u$ - control value; $y_{p}-$ process output; $y_{m}-$ model output; $e=y_{p}-y_{m}-$ output error. 


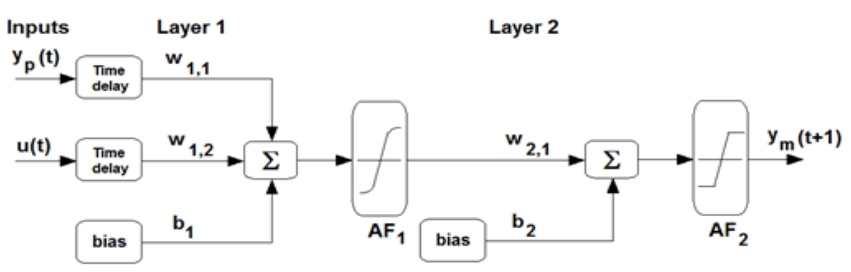

Fig.2. Neural model of the process.

Notations in figure 2 are: $u(t)-$ control value at time $t ; y_{p}(t)$ - process output at time $t ; y_{m}(t+1)$ - model output at time $t+1$ (control estimate; $w_{i, j}$ - input weight for input $j$ in layer $i ; b_{k}$ - bias for layer $k ; A F_{h}$ - activation function for layer $h$.

Operation phase. The neural model generates estimations of the process response in a certain time horizon. These estimations are used in an optimization algorithm in order to find those values of of the control values which minimize the criterion function:

$J=\sum_{j=N_{1}}^{N_{2}}\left(y_{r}(t+j)-y_{m}(t+j)\right)^{2}+\rho \sum_{j=1}^{N_{u}}\left(u^{\prime}(t+j-1)-u^{\prime}(t+j-2)\right)^{2}$

in which:

- $\quad N_{l}, N_{2}$ and $N_{u}$ defines time horizons in which the values of control error are evaluated;

$u$ ' - test control signal;

$y_{r}$ - desired response;

$y_{m}$ - neural model response;

- $\quad \rho$ - defines the contribution of control value variation in previous time horizons.

Figure 3 presents the controller which is composed by the neural model and the optimization bloc. The optimization bloc defines which values $u$ ' minimizes the criteria function $\mathbf{J}$. Once the optimal value $u$ is found this is used as control value of the process.

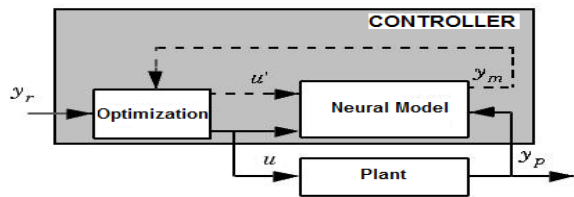

Fig.3. Controlled process diagram.

As the described algorithm shows, soft computing methods can successfully be combined with usual control methods, in this case MPC. There are several studies regarding combination of MPC and neural methods as for example in [7] where the problem of controlling a Heating Ventilation and Air Conditioning (HVAC) system with the purpose of achieving a desired thermal comfort level and energy savings is dealt with. A discrete model-based predictive control methodology is applied, consisting of three major components: the predictive models, implemented by radial basis function neural networks identified by means of a multiobjective genetic algorithm; the cost function that will be optimized to minimize energy consumption and maintain thermal comfort; and the optimization method, a discrete branch and bound approach. Experimental results obtained within different rooms in a certain building demonstrated the feasibility and performance of the approach. Energy savings resulting from the application of the method were estimated to be greater than $50 \%$. Other researchers are using ANN to build complex models as for example in [8] where the authors trained such a neural network to learn and predict the indoor temperature of rooms with the different experimental data. In this case the researchers seek to achieve thermal comfort in a building by applying different passive cooling techniques. The presented inputs were the outside temperature, relative humidity, solar intensity and wind speed. The network output was indoor temperature. The advantages of this approach are the computational speed, simplicity, adaptive learning, selforganization and real time operation.

\subsection{Fuzzy logic}

Fuzzy logic is another widely used soft computing method to enhance control in Intelligent Building applications. This method is used in different situations and on different levels of complex control system. For example at a specific level in [9] there is proposed a fuzzy rule-based system that estimates the next duty cycle of sensor batteries, which are used in IB and smart home devices. The structure presented in [10] consists of one coordinator and five fuzzy logic controllers. Type-1 FS models are chosen to characterize the concepts of thermal comfort, luminance and carbon dioxide concentration. The tuning of parameters of the fuzzy logic controller is made by genetic algorithms (GAs). The use of intelligent agents is also the subject in [11] where these agents are based on type- 2 fuzzy systems, able to handle the different sources of uncertainty and imprecision in ubiquitous computing environments (UCEs). The study presented in [12] shows that fuzzy logic is used not only in complex control systems but also to adapt devices to human users or to develop decision models [13]. The authors of [17] propose the concept of emerging and adaptive fuzzy logic based behaviors which are based on dynamically created activity spheres to autonomously learn and adapt to the requirements of the human users in environments which are populated with a large number of potentially heterogeneous devices (different manufacturers, different descriptions of properties, etc.). In [13] the authors develop a decision model for managing uncertainty and imprecision of green building project evaluation during the design process. The evaluation is performed by a procedure based on a combination of fuzzy analytic hierarchy process and the Technique for Order of Preference by Similarity to Ideal Solution (TOPSIS) which is a multi-criteria decision analysis method. In addition to energy saving and user comfort, an important field in which the smart home and intelligent building concept is the assisted living where disabled or elder person's life can be substantially enhanced by the benefits of these concepts. This is stated in [14] where is shown that Smart Homes are intelligent spaces that contain resources to collect information about user's 
activities and ease the assisted living. The authors focus on abnormal behavior detection which is remarked as one of the most challenging application fields in this research area, due to its possibilities for assisting elders or people with special needs. These systems help to maintain people's independence, enhancing their personal comfort and safety and delaying the process of moving to a nursing home. A new approach is proposed for the behavior recognition problem based on Learning Automata and fuzzy temporal windows. The system learns the normal behaviors, and uses that knowledge to recognize normal, abnormal human activities and changes in human habits in real time.

\subsection{Swarm intelligence}

In the study presented in [15], to effectively manage the energy consumption and occupants' comfort, a multi-agent based control framework is proposed for smart building applications. The energy consumption and the overall comfort level are considered as two control objectives. Two multiobjective optimization methods including multi-objective particle swarm optimization (MOPSO) and weighted aggregation are utilized to generate the Pareto fronts which are made up of Pareto-optimal solutions.

Particle swarm optimization is also used in [16] where a multi-zone building model is built for developing an effective energy management scheme. This study proposes a multi-agent control system coupled with an intelligent optimizer for intelligent building control. Particle swarm optimization (PSO) is utilized to optimize the building energy management by enhancing the intelligence of the multi-zone building during its operations.

\section{SUMMARY}

In this paper we synthetized a small but hopefully representative part of the applications of soft computing methods in the field of intelligent buildings, smart homes, ambient ecologies and ubiquitous computing. We tried to highlight the advantages of these methods in solving difficult control problems, analyze and modelling of human behavior in order to implement energy saving control solutions besides accounting for user comfort. Our study is aimed to help to gather information which will be helpful in developing new control strategies in this field.

\section{ACKNOWLEDGEMENTS}

This work was supported by the European Union and cofinanced by the States of Hungary and Romania in the framework of cross-border cooperation program CBC-HURO, 0802/155_AF, "Romanian-Hungarian R\&D Platform for intelligent building research projects support ",acronym "Intelbuild" project.

\section{References}

[1] Johnny Wong, Heng Li, Jenkin Lai, Evaluating the system intelligence of the intelligent building systems, Part 1:
Development of key intelligent indicators and conceptual analytical framework, Automation in Construction 17 (2008) 284302.

[2] Ayesha Kashif, Stéphane Ploix, Julie Dugdale, Xuan Hoa Binh Le, Simulating the dynamics of occupant behavior for power management in residential buildings, Energy and Buildings 56 (2013) 85-93.

[3] Laura Klein, Jun-young Kwak, Geoffrey Kavulya, Farrokh Jazizadeh, Burcin Becerik-Gerber, Pradeep Varakantham, Milind Tambe, Coordinating occupant behavior for building energy and comfort management using multi-agent systems, Automation in Construction 22 (2012) 525-536.

[4] Z.B. Zhao, W.S. Xu, D.Z. Cheng, User behavior detection framework based on NBP for energy efficiency, Automation in Construction 26 (2012) 69-76.

[5] Tuan Anh Nguyen, Marco Aiello, Energy intelligent buildings based on user activity: A survey, Energy and Buildings 56 (2013) 244-257.

[6] Jiri Cigler, Pavel Tomasko, Jan Siroky, BuildingLAB: A tool to analyze performance of model predictive controllers for buildings, Energy and Buildings 57 (2013) 34-41.

[7] P.M. Ferreira,*, A.E. Ruanoa, S. Silva, E.Z.E. Conceicao, Neural networks based predictive control for thermal comfort and energy savings in public buildings, Energy and Buildings 55 (2012) 238251.

[8] Shrikant Pandey, D.A. Hindoliya, Ritu Mod, Artificial neural networks for predicting indoor temperature using roof passive cooling techniques in buildings in different climatic conditions, Applied Soft Computing 12 (2012) 1214-1226.

[9] J.C. Cuevas-Martinez, J. Canada-Bago, J.A. Fernandez-Prieto, M.A. Gadeo-Martos, Knowledge-based duty cycle estimation in wireless sensor networks: Application for sound pressure monitoring, Applied Soft Computing 13 (2013) 967-980, 2012 Elsevier B.V.

[10] A.I. Dounis, P. Tiropanis, A. Argiriou, A. Diamantis, Intelligent control system for reconciliation of the energy savings with comfort in buildings using soft computing techniques, Energy and Buildings 43 (2011) 66-74, 2010 Elsevier B.V.

[11] Faiyaz Doctor, Hani Hagras, Victor Callaghan, A type-2 fuzzy embedded agent to realize ambient intelligence in ubiquitous computing environments, Information Sciences 171 (2005) 309334, 2004 Elsevier Inc.

[12] Sahin Yildirim; Husi Géza: Artifical neural network applications in engineering problems Oradea: Editura Universitatii din Oradea, 2010. 85 p. ISBN: $978-606-10-0252-8$

[13] Che-Wei Chang, Develop a ranking algorithm for the green building project, Qual Quant, DOI 10.1007/s11135-012-9812-2, Springer Science+Business Media Dordrecht 2012.

[14] M. Ros, M.P. Cuéllar, M. Delgado, A. Vila, Online recognition of human activities and adaptation to habit changes by means of learning automata and fuzzy temporal windows, Information Sciences 220 (2013) 86-101, 2011 Elsevier Inc.

[15] Rui Yang, Lingfeng Wang, Multi-objective optimization for decision-making of energy and comfort management in building automation and control, Sustainable Cities and Society 2 (2012) 17.

[16] Rui Yang, Lingfeng Wang, Multi-zone building energy management using intelligent control and Optimization, Sustainable Cities and Society 6 (2013) 16-21.

[17] Christian Wagner, Christos Goumopoulos, Hani Hagras, Emerging and adaptive fuzzy logic based behaviors in activity sphere centered ambient ecologies, Pervasive and Mobile Computing 8 (2012) 500-521, 2011 Elsevier B 